\title{
INVARIANTIVE CHARACTERIZATIONS OF LINEAR ALGEBRAS WITH THE ASSOCIATIVE LAW NOT ASSUMED*
}

BY

\section{CYRUS COLTON MACDUFFEE}

\section{INTRODUCTION}

We consider linear algebras in which neither the commutative nor the associative law of multiplication is assumed, and whose coördinates and constants of multiplication are numbers of a general field $F$. A rational integral invariant, or covariant, is a rational integral function of the constants of multiplication, or of the constants of multiplication and the coördinates of the general number, which has the invariantive property under the total group of linear homogeneous transformations on the units. If an invariantive function also actually involves the units, it has been called a vector covariant by Professor O. C. Hazlett, $\dagger$ who shows that every rational integral vector covariant can be obtained as a covariant of the general number of the algebra and a fundamental set of ordinary covariants.

In Section II of this article, it is shown how vector covariants may be formed directly from the constants of multiplication without assuming the knowledge of any ordinary covariants or invariants. To do this, the notion is introduced of a hypercomplex determinant whose elements obey neither the associative nor the commutative law of multiplication, and a few simple properties of such hypercomplex determinants are derived. From the vector covariants and the characteristic determinants of the algebra, ordinary relative invariants may easily be found.

In Section III the linear algebra in three units, one of which is a principle unit, is considered. Invariants and covariants of the algebra are calculated by the method of Section II, and a set of ten of these functions is shown to form a complete system of invariants and covariants from the standpoint of Lie.

In Section IV it is shown that for the example of Section III the arithmetic invariant denoting the rank can be replaced by a rational integral covariant. The generic case is defined as the case for which certain three covariants are

* Presented to the Society, March 25, 1921.

† These Tr a n s a c ti o n s, vol. 19 (1918), p. 408. 
different from zero, and this case is reduced by rational transformations in the general field to a canonical form. The parameters of the canonical form are characterized by invariants.

\section{INVARIANTS AND VECTOR COVARIANTS}

1. Notations. Consider the general linear algebra in $n$ units whose multiplication table is given by

$$
e_{i} e_{j}=\sum_{k=1}^{k=n} \gamma_{i j k} e_{k} \quad(i, j=1, \ldots, n),
$$

and whose general number is $x=\Sigma_{i=1}^{i=n} x_{i} e_{i}$, where the $\gamma_{i j k}$ and $x_{i}$ are numbers of a general field $F$. Consider the transformation of units

$$
T: e_{i}{ }^{\prime}=\sum_{j=1}^{j=n} \alpha_{i j} e_{j} \quad D \equiv\left|\alpha_{i j}\right| \neq 0 \quad(i=1, \ldots, n),
$$

also with coefficients $\alpha_{i j}$ in $F$. This transformation $T$ induces upon the constants $\gamma_{i j k}$ of multiplication and the coördinates $x_{i}$ a transformation $S$ which carries $\gamma_{i j k}$ into $\gamma_{i j k}^{\prime}$ and $x_{i}$ into $x_{i}^{\prime}$ in such a way that

$$
e_{i}^{\prime} e_{j}^{\prime}=\sum_{k=1}^{k=n} \gamma_{i j k}^{\prime} e_{k}^{\prime}
$$

and

$$
\sum_{j=1}^{j=n} x_{j} e_{j}=x=x^{\prime}=\sum_{i=1}^{i=n} x_{i}^{\prime} e_{i}^{\prime}
$$

According to Professor Hazlett, ${ }^{*}$ a vector covariant is defined to be a function of the units and the constants of multiplication and the coordinates of the form

$$
v\left(\gamma_{i j k} ; x_{r} ; e_{s}\right) \quad(i, j, k, r, s=1, \ldots, n),
$$

such that, under a transformation $T$ of the units and its induced transformation $S$, there exists a relation of the form

$$
v^{\prime} \equiv v\left(\gamma_{i j k}^{\prime} ; x_{r}^{\prime} ; e_{s}^{\prime}\right)=D^{\mu} v\left(\gamma_{i j k} ; x_{r} ; e_{s}\right) \quad(i, j, k, r, s=1, \ldots, n)
$$

The integer $\mu$ is called the weight of the vector covariant $v$.

As Miss Hazlett noted, $\nmid$ it follows readily from (1) and (3) that every vector covariant is expressible linearly in the units, i. e.,

$$
v^{\prime}=\sum_{i=1}^{i=n} v_{i}^{\prime} e_{i}^{\prime}, \quad v=\sum_{i=1}^{i=n} v_{i} e_{i},
$$

where $v_{i}^{\prime}$ is the same function of the $\gamma_{i j k}^{\prime}$ and $\dot{x}_{r}^{\prime}$ that $v_{i}$ is of the $\gamma_{i j k}$ and the $x_{r}$.

* These Tr a n s a c t i on s, vol. 19 (1918), p. 408.

† Ibid., p. $\$ 16$. 
2. Theorem 1. The coelficients $v_{i}$ of every vector covariant of weight $\mu$ expressed in the form $v=\sum_{i=1}^{i=n} v_{i} e_{i}$ are transformed cogrediently apart from the factor $D^{-\mu}$ with the coordinates $x_{i}$ of the general number $x=\sum_{i=1}^{i=n} x_{i} e_{i}$ under a linear transformation of determinant $D$.

From (5) and (6) we have

$$
\sum_{i=1}^{i=n} v_{i}^{\prime} e_{i}^{\prime}=D^{\mu} \sum_{j=1}^{j=n} v_{j} e_{j}
$$

Making use of (2), we find that

$$
\begin{aligned}
\sum_{j=1}^{j=n} v_{j} e_{j} & =D^{-\mu} \sum_{i=1}^{i=n} v_{i}^{\prime} \sum_{j=1}^{j=n} \alpha_{i j} e_{j} \\
& =\sum_{j=1}^{j=n} D^{-\mu} \sum_{i=1}^{-1=n} v_{i}^{\prime} \alpha_{i j} e_{j} .
\end{aligned}
$$

On account of the linear independence of the units $e_{j}$, which occur only where shown explicitly,

$$
v_{j,}^{*}=D^{-\mu} \sum_{i=1}^{i=n} v_{i}^{\prime} \alpha_{i j} \quad(j=1, \ldots, n) .
$$

Similarly from (4) and (2) we have

$$
x_{j}=\sum_{i=1}^{i=n} x_{i}^{\prime} \alpha_{i j} \quad(j=1, \ldots, n) .
$$

Comparing (7) with (8), we see that the theorem is proved.

3. Hypercomplex determinants.* On account of the fact that multiplication is usually neither commutative nor associative, a determinant whose elements are hypercomplex numbers must be defined more precisely than a determinant whose elements are ordinary numbers. We define the general hypercomplex determinant of the $n$th order

$$
\begin{aligned}
& D=\left|\begin{array}{ccc}
a_{11} & \ldots & a_{1 n} \\
\cdot & \cdot & \cdot \\
a_{n 1} & \ldots & a_{n n}
\end{array}\right| \\
& {\left[a_{11} a_{22} \ldots a_{n n}\right]}
\end{aligned}
$$

to be the sum of $n$ ! terms of the type

$$
(-1)^{k}\left[a_{1 i_{1}} a_{2 i_{2}} \ldots a_{n i_{n}}\right]
$$

in which $i_{1}, i_{2}, \ldots, i_{n}$ is an arrangement of $1,2, \ldots, n$ derived from the natural order by $k$ interchanges. The first subscripts must occur in their natural order in every term. The brackets indicate that the method of grouping the factors is arbitrary, but the same method is to be used in each term. A particular

* Determinants whose elements are quaternions and hence associative but not commutative were considered by Cayley, On certain results relating to quaternions, The $\mathrm{Ph}$ ilos o $\mathrm{ph}$ ic a $1 \mathrm{M}$ a g a z i n e, vol. 26 (1845) pp. 141-145; and also by C. J. Joly, second edition of Hamilton's Elements of Quaternions, vol. 2, Appendix 1, p. 361. 
hypercomplex determinant of the $n$th order is obtained by replacing the brackets inclosing the leading term by a particular grouping of that term, in which case it is understood that every term of the expansion is to be grouped in that way. Thus there are as many particular hypercomplex determinants of the $n$th order as there are ways of grouping $n$ ordered factors.

4. Lemma 1. Every hypercomplex determinant merely changes sign upon the interchange of any two columns.

Consider the determinant (9). Its terms may be arranged in $\frac{1}{2}(n !)$ pairs of the type

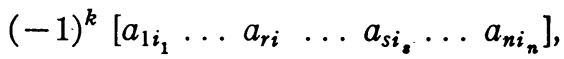

$$
\begin{aligned}
& (-1)^{k+1}\left[a_{1 i_{1}} \ldots a_{r i_{s}} \ldots a_{s i_{r}} \ldots a_{n i_{n}}\right] \text {, }
\end{aligned}
$$

where the two.terms in each pair differ only in having their $i_{r}$ and $i_{s}$ subscripts interchanged. Since each is obtainable from the other by one interchange of subscripts, they are opposite in sign. Let us denote $D$ with its $r$ th and $s$ th columns interchanged by $D^{\prime}$. Evidently this interchange leaves the first subscripts in their natural order but interchanges the second subscripts $i_{r}$ and $i_{s}$ wherever they occur, and so interchanges the absolute values of the terms in each pair (10). Thus every term in $D$ with its sign changed is equal to a term of $D^{\prime}$ and vice versa. Then $D^{\prime}=-D$. This argument depends in no way upon the manner of grouping, so the lemma holds for all hypercomplex determinants.

No analogous theorem exists concerning the interchange of two rows.

Lemma 2. Any hypercomplex determinant two of whose columns are identical is «ero.

For by the interchange of the two identical columns the determinant is unaltered, and yet changes in sign.

5. Lemma 3. A hypercomplex determinant the elements of whose jth column are binomials $a_{i j}+b_{i j}$ is equal to the sum of two determinants identical with the first except that the jth column of the one is composed of the $a_{i j}$ while the jth column of the other is composed of the $b_{i j}$. The corresponding theorem holds for rows.

Let us set

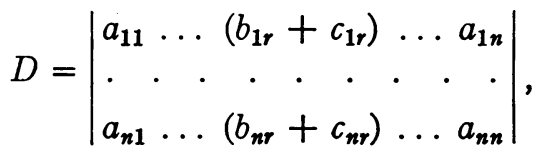

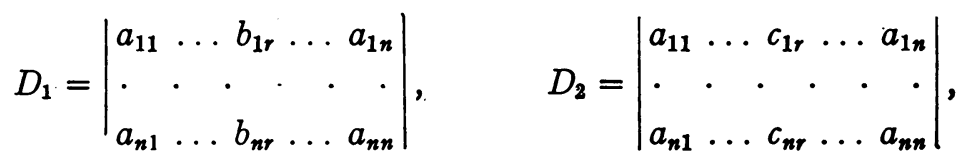


where it is understood that the manner of grouping is arbitrary, but the same manner must be used consistently in every term of each determinant. It follows from the distributive law that

$$
a_{1 i_{1}} \ldots\left(b_{s i_{\text {s }}}+c_{s i_{\mathrm{s}}}\right) \ldots a_{n i_{n}}=a_{1 i_{1}} \ldots b_{s i_{8}} \ldots a_{n i_{n}}+a_{1 i_{1}} \ldots c_{s i_{8}} \ldots a_{n i_{n}}
$$

for all values of the subscripts and for all methods of grouping. It holds in particular for $i_{s}=r$ and $s=1, \ldots, n$ where $r$ is fixed. Let $k$ denote the number of interchanges necessary to obtain the order $i_{1}, \ldots, i_{n}$ from the natural order $1, \ldots, n$. Then, multiplying $(11)$ by $(-1)^{k}$ and summing for $i_{1}, \ldots, i_{n}$, we have

$$
D=D_{1}+D_{2}
$$

which was to be proved.

We prove the corresponding theorem for rows by noting that (11) holds when $s$ is fixed and $i_{s}$ ranges over the values $1, \ldots, n$, and summing as before.

6. Theorem 2. The determinant

$$
V \equiv\left|\begin{array}{ccc}
e_{1} & \ldots & e_{n} \\
\cdot & \cdot & \cdot \\
e_{1} & \ldots & e_{n}
\end{array}\right|
$$

vector covariant of weight 1 of the linear algebra in $n$ units $e_{1}, \ldots, e_{n}$ for every manner of grouping.

We set

$$
V^{\prime} \equiv\left|\begin{array}{ccc}
e_{1}^{\prime} & \ldots & e_{n}^{\prime} \\
\cdot & \cdot & \cdot \\
e_{1}^{\prime} & \ldots & e_{n}^{\prime}
\end{array}\right|
$$

Under transformation (2) this becomes

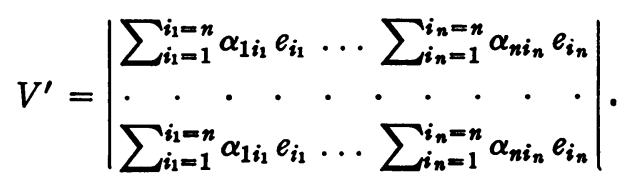

From Lemma 3 we see that

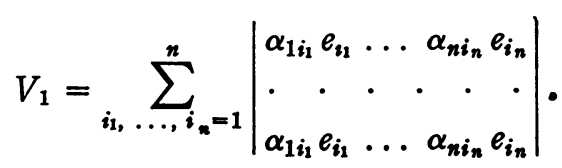

By Lemma 2 such of these determinants as have two identical columns are zero. Moreover the $\alpha$ 's are numbers of the field $F$. Hence we may restrict the $i_{1}$, $\ldots, i_{n}$ to sets of $n$ distinct values and write 


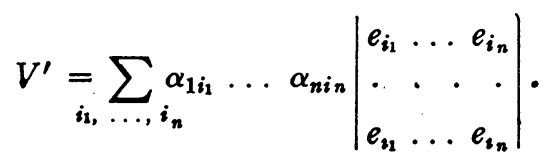

Let $k$ denote the number of interchanges necessary to produce the order $i_{1}$, $\ldots, i_{n}$ from the natural order of the integers $1, \ldots, n$. By Lemma 1 the above determinant changes sign with each such interchange, that is with each interchange of two columns. Then

$$
V^{\prime}=\sum_{i_{1}, \ldots, i_{n}}(-1)^{k} \alpha_{1 i_{1}} \ldots \alpha_{n i_{n}}\left|\begin{array}{ccc}
e_{1} & \ldots & e_{n} \\
\cdot & . & \cdot \\
e_{1} & \ldots & e_{n}
\end{array}\right|
$$

The above sum is exactly the determinant $D$ of the transformation (2). Hence, as stated,

$$
V^{\prime}=D V
$$

7. Rational integral invariants. In the general linear algebra in $n$ units the right- and left-hand characteristic determinants*

$$
\begin{aligned}
\delta(\omega) & =\left|\sum_{i=1}^{b=n} \gamma_{i j k} x_{i}-\delta_{j k} \omega\right|, \\
\delta^{\prime}(\omega) & =\left|\sum_{j=1}^{j=n} \gamma_{i j k} x_{j}-\delta_{i k} \omega\right| \quad(i, j, k=1, \ldots, n),
\end{aligned}
$$

and the coefficients of the powwers of $\omega$ therein are absolute covariants under the general group of homogeneous linear transformations on the units. In view of Theorem 2 , there are conceivably as many vector covariants of weight 1 as there are ways of grouping $n$ ordered factors, but it may happen in certain algebras that a smaller number results. Thus for commutative algebras they all vanish and for associative algebras they become identical. By means of the multiplication table (1) each such vector covariant can be expressed linearly in terms of the units, i. e., in the form (6). Suppose that $p$ of them are linearly independent. By Theorem 1 the coefficients $v_{i}$ are transformed cogrediently apart from the factor $D^{-1}$ with the coördinates $x_{i}$ of the general number. Therefore if these coefficients $v_{i}$ be inserted in place of the $x_{i}$ in a homogeneous absolute invariant of degree $r$, there results a relative invariant of the linear algebra of weight $r$. This method yields $2 n p$ relative invariants.

\section{A LIE COMPLETE SYSTEM OF INVARIANTS AND COVARIANTS FOR \\ A TERNARY ALGEBRA}

8. Foreword. It will be shown that for the algebra with three units, one of which is a principal unit, the invariants formed by the method of the preceding section together with those obtained from the characteristic determinants by

* L. E. Dickson, Linear Algebras, Cambridge Tract No. 16 (Cambridge, 1914), p. 17 and p. 20. 
the methods of the theory of invariants of forms constitute a complete system of invariants and covariants from the standpoint of Lie. In fact, if all the invariants obtainable by both methods are taken, there is considerable redundance. A set of the simpler ones is shown to be composed of functionally independent invariants.

9. The finite transformations. In the linear algebra in three units $1, e_{1}, e_{2}$, where 1 is a principal unit, the general number is of the form

$$
x=x_{0}+x_{1} e_{1}+x_{2} e_{2}
$$

where $x_{0}, x_{1}, x_{2}$ are numbers of a field $F$. The multiplication table may be taken to be

$$
\begin{aligned}
e_{1}^{2} & =a_{0}+a_{1} e_{2}+a_{2} e_{2}, \\
e_{2}^{2} & =b_{0}+b_{1} e_{1}+b_{2} e_{2}, \\
e_{1} e_{2} & =c_{0}+c_{1} e_{1}+c_{2} e_{2} \\
e_{2} e_{1} & =d_{0}+d_{1} e_{1}+d_{2} e_{2},
\end{aligned}
$$

where the coefficients are numbers of $F$. By applying the linear transformation of units

$$
\begin{aligned}
& e_{1}^{\prime}=\alpha_{0}+\alpha_{1} e_{1}+\alpha_{2} e_{2}, \\
& e_{2}^{\prime}=\beta_{0}+\beta_{1} e_{1}+\beta_{2} e_{2},
\end{aligned}
$$

where the coefficients are numbers of $F$ such that $D \equiv \alpha_{1} \beta_{2}-\alpha_{2} \beta_{1}$ is different from zero, the principal unit is left invariant and the general number and multiplication table become (13) and (14) respectively with each letter primed. That is, the transformation (15) of units induces upon the coefficients the following transformation:

$$
\begin{aligned}
& x_{0}^{\prime}=x_{0}+D^{-1}\left(\alpha_{2} \beta_{0}-\alpha_{0} \beta_{2}\right) x_{1}+D^{-1}\left(\alpha_{0} \beta_{1}-\alpha_{1} \beta_{0}\right) x_{2}, \\
& x_{1}^{\prime}=D^{-1} \beta_{2} x_{1}-D^{-1} \beta_{1} x_{2}, \\
& x_{2}^{\prime}=-D^{-1} \alpha_{2} x_{1}+D^{-1} \alpha_{1} x_{2}, \\
& a_{0}^{\prime}=A+D^{-1}\left(\alpha_{2} \beta_{0}-\alpha_{0} \beta_{2}\right) B+D^{-1}\left(\alpha_{0} \beta_{1}-\alpha_{1} \beta_{0}\right) C, \\
& a_{1}^{\prime}=D^{-1} \beta_{2} B-D^{-1} \beta_{1} C, \\
& a_{2}^{\prime}=-D^{-1} \alpha_{2} B+D^{-1} \alpha_{1} C, \\
& b_{0}^{\prime}=E+D^{-1}\left(\alpha_{2} \beta_{0}-\alpha_{0} \beta_{2}\right) F+D^{-1}\left(\alpha_{0} \beta_{1}-\alpha_{1} \beta_{0}\right) G, \\
& b_{1}^{\prime}=D^{-1} \beta_{2} F-D^{-1} \beta_{1} G \\
& b_{2}^{\prime}=-D^{-1} \alpha_{2} F+D^{-1} \alpha_{1} G \\
& c_{0}^{\prime}=H+D^{-1}\left(\alpha_{2} \beta_{0}-\alpha_{0} \beta_{2}\right) I+D^{-1}\left(\alpha_{0} \beta_{1}-\alpha_{1} \beta_{0}\right) J, \\
& c_{1}^{\prime}=D^{-1} \beta_{2} I-D^{-1} \beta_{1} J \\
& c_{2}^{\prime}=-D^{-1} \alpha_{2} I+D^{-1} \alpha_{1} J \\
& d_{0}^{\prime}=K+D^{-1}\left(\alpha_{2} \beta_{0}-\alpha_{0} \beta_{2}\right) L+D^{-1}\left(\alpha_{0} \beta_{1}-\alpha_{1} \beta_{0}\right) M, \\
& d_{1}^{\prime}=D^{-1} \beta_{2} L-D^{-1} \beta_{1} M, \\
& d_{2}^{\prime}=-D^{-1} \alpha_{2} L+D^{-1} \alpha_{1} M,
\end{aligned}
$$


where

$$
\begin{aligned}
& A \equiv \alpha_{0}^{2}+\alpha_{1}^{2} a_{0}+\alpha_{2}^{2} b_{0}+\alpha_{1} \alpha_{2} c_{0}+\alpha_{1} \alpha_{2} d_{0}, \\
& B \equiv \alpha_{1}^{2} a_{1}+\alpha_{2}^{2} b_{1}+2 \alpha_{0} \alpha_{1}+\alpha_{1} \alpha_{2} c_{1}+\alpha_{1} \alpha_{2} d_{1}, \\
& C \equiv \alpha_{1}^{2} a_{2}+\alpha_{2}^{2} b_{2}+2 \alpha_{0} \alpha_{2}+\alpha_{1} \alpha_{2} c_{2}+\alpha_{1} \alpha_{2} d_{2}, \\
& E \equiv \beta_{0}^{2}+\beta_{1}^{2} a_{0}+\beta_{2}^{2} b_{0}+\beta_{1} \beta_{2} c_{0}+\beta_{1} \beta_{2} d_{0}, \\
& F \equiv \beta_{1}^{2} a_{1}+\beta_{2}^{2} b_{1}+2 \beta_{0} \beta_{1}+\beta_{1} \beta_{2} c_{1}+\beta_{1} \beta_{2} d_{1}, \\
& G \equiv \beta_{1}^{2} a_{2}+\beta_{2}^{2} b_{2}+2 \beta_{0} \beta_{2}+\beta_{1} \beta_{2} c_{2}+\beta_{1} \beta_{2} d_{2}, \\
& H \equiv \alpha_{0} \beta_{0}+\alpha_{1} \beta_{1} a_{0}+\alpha_{2} \beta_{2} b_{0}+\alpha_{1} \beta_{2} c_{0}+\alpha_{2} \beta_{1} d_{0}, \\
& I \equiv \alpha_{1} \beta_{0}+\alpha_{0} \beta_{1}+\alpha_{1} \beta_{1} a_{1}+\alpha_{2} \beta_{2} b_{1}+\alpha_{1} \beta_{2} c_{1}+\alpha_{2} \beta_{1} d_{1}, \\
& J \equiv \alpha_{2} \beta_{0}+\alpha_{0} \beta_{2}+\alpha_{1} \beta_{1} a_{2}+\alpha_{2} \beta_{2} b_{2}+\alpha_{1} \beta_{2} c_{2}+\alpha_{2} \beta_{1} d_{2}, \\
& K \equiv \alpha_{0} \beta_{0}+\alpha_{1} \beta_{1} a_{0}+\alpha_{2} \beta_{2} b_{0}+\alpha_{2} \beta_{1} c_{0}+\alpha_{1} \beta_{2} d_{0}, \\
& L \equiv \alpha_{0} \beta_{1}+\alpha_{1} \beta_{0}+\alpha_{1} \beta_{1} a_{1}+\alpha_{2} \beta_{2} b_{1}+\alpha_{2} \beta_{1} c_{1}+\alpha_{1} \beta_{2} d_{1}, \\
& M \equiv \alpha_{0} \beta_{2}+\alpha_{2} \beta_{0}+\alpha_{1} \beta_{1} a_{2}+\alpha_{2} \beta_{2} b_{2}+\alpha_{2} \beta_{1} c_{2}+\alpha_{1} \beta_{2} d_{2} .
\end{aligned}
$$

10. The Lie group. The generators* of the infinitesimal transformation corresponding to the finite transformation (16) are found to consist of six partial differential equations which it is not necessary to give here in detail. The first term of each equation involves only the $x_{i}$, and is the only term in which the $x_{i}$ occur. Since these equations are generators of an infinitesimal group corresponding to the finite group of transformations (16), they form a fortiori a complete system of partial differential equations. The six equations in fifteen variables have nine functionally independent solutions, and these solutions form a complete set of absolute invariants and covariants of the linear algebra (14).

Moreover, if the terms involving $x_{0}, x_{1}, x_{2}$ in these equations be deleted, there results another complete system of partial differential equations, the generators of the group corresponding to the group of transformations in (16) on the constants of multiplication only. This system has six functionally independent solutions, the six absolute invariants of the linear algebra. They are six of the nine solutions of the first complete system. We see then that there are exactly six absolute invariants and three absolute covariants in a complete system of invariants and covariants for the algebra. There are then no more than seven functionally independent relative invariants. A complete system will be exhibited of three absolute covariants and seven relative invariants whose jacobian does not vanish identically.

11. Invariants of the characteristic determinants. The right- and left-hand characteristic determinants (12) become

$$
\begin{gathered}
\delta(\omega)=-\omega^{3}+\Lambda \omega^{2}-\Gamma \omega+\Delta, \\
\delta^{\prime}(\omega)=-\omega^{3}+\Lambda^{\prime} \omega^{2}-\Gamma^{\prime} \omega+\Delta^{\prime}
\end{gathered}
$$

* Lie-Scheffers, Vorlesungen über continuierliche Gruppen, Leipzig, 1893, p. 716, et seq. 
where

$$
\begin{aligned}
& \Lambda=3 x_{0}+\left(a_{1}+c_{2}\right) x_{1}+\left(d_{1}+b_{2}\right) x_{2} \\
& \Gamma=3 x_{0}^{2}+\left(a_{1} c_{2}-a_{2} c_{1}-a_{0}\right) x_{1}^{2}+\left(b_{2} d_{1}-b_{1} d_{2}-b_{0}\right) x_{2}^{2}+\left(2 a_{1}\right. \\
& \left.+2 c_{2}\right) x_{0} x_{1}+\left(2 b_{2}+2 d_{1}\right) x_{0} x_{2}+\left(a_{1} b_{2}-a_{2} b_{1}+c_{2} d_{1}-c_{1} d_{2}-d_{0}-c_{0}\right) x_{1} x_{2} \\
& \quad \Delta=x_{0}^{3}+\left(a_{2} c_{0}-a_{0} c_{2}\right) x_{1}^{3}+\left(b_{1} d_{0}-b_{0} d_{1}\right) x_{2}{ }^{3}+\left(a_{1} c_{2}-a_{2} c_{1}\right. \\
& \left.-a_{0}\right) x_{0} x_{1}^{2}+\left(a_{1}+c_{2}\right) x_{0}^{2} x_{1}+\left(b_{2}+d_{1}\right) x_{0} x_{2}+\left(b_{2} d_{1}-b_{1} d_{2}-b_{0}\right) x_{0} x_{2}^{2} \\
& +\left(c_{0} d_{2}-c_{2} d_{0}+a_{2} b_{0}-a_{0} b_{2}+a_{0} c_{1}-a_{1} c_{0}\right) x_{1}^{2} x_{2}+\left(b_{0} d_{2}-b_{2} d_{0}+a_{0} b_{1}\right. \\
& \left.-a_{1} b_{0}+c_{1} d_{0}-c_{0} d_{1}\right) x_{1} x_{2}^{2}+\left(c_{2} d_{1}-c_{1} d_{2}+a_{1} b_{2}-a_{2} b_{1}-c_{0}\right. \\
& \left.-d_{0}\right) x_{0} x_{1} x_{2},
\end{aligned}
$$

and $\Lambda^{\prime}, \Gamma^{\prime}, \Delta^{\prime}$ are obtained from $\Lambda, \Gamma, \Delta$ respectively by interchanging $c_{i}$ and $d_{i}$, for $i=0,1,2$. Each of these six expressions satisfies the equations of $\$ 10$ and is an absolute covariant of the algebra.

The invariants of the six ternary forms (17) are invariants of the algebra. $\Lambda$ and $\Lambda^{\prime}$ have no invariants. $\Gamma$ and $\Gamma^{\prime}$ have one invariant each, their hessians. The ternary cubic forms $\Delta$ and $\Delta^{\prime}$ each have two relative invariants, the $S$ and $T$ of Aronhold.* But only six of the seven invariants have been accounted for by this means, even if all six should prove to be independent. Thus it is evident that the invariants obtainable as the invariants of the coefficients of the characteristic determinants are not sufficient to form a complete system.

12. Additional invariants by the method of Section II. By Theorem 2 of $\$ 6$, the hypercomplex determinant

$$
V \equiv\left|\begin{array}{lll}
1 & e_{1} & e_{2} \\
1 & e_{1} & e_{2} \\
1 & e_{1} & e_{2}
\end{array}\right|=e_{1} e_{2}-e_{2} e_{1}
$$

is a vector covariant of weight 1 . By the multiplication table (14) this can be expressed as

$$
V=\left(c_{0}-d_{0}\right)+\left(c_{1}-d_{1}\right) e_{1}+\left(c_{2}-d_{2}\right) e_{2} \text {. }
$$

Now by Theorem $1, \S 2$, the coefficients of the units in (18) are transformed cogrediently apart from the factor $D^{-1}$ with the coefficients $x_{0}, x_{1}, x_{2}$ of the general number, and hence when substituted for these variables in (17) give relative invariants of the algebra. $\dagger$ Thus we have

$$
\begin{aligned}
& \Lambda_{1}=3\left(c_{0}-d_{0}\right)+\left(a_{1}+c_{2}\right)\left(c_{1}-d_{1}\right)+\left(d_{1}+b_{2}\right)\left(c_{2}-d_{2}\right), \\
& \Lambda_{1}^{\prime}=3\left(c_{0}-d_{0}\right)+\left(a_{1}+d_{2}\right)\left(c_{1}-d_{1}\right)+\left(c_{1}+b_{2}\right)\left(c_{2}-d_{2}\right), \\
& \Gamma_{2}=3\left(c_{0}-d_{0}\right)^{2}+\left(a_{1} c_{2}-a_{2} c_{1}-a_{0}\right)\left(c_{1}-d_{1}\right)^{2}+\cdots, \\
& \Gamma_{2}^{\prime}=3\left(c_{0}-d_{0}\right)^{2}+\left(a_{1} d_{2}-a_{2} d_{1}-a_{0}\right)\left(c_{1}-d_{1}\right)^{2}+\cdots, \\
& \Delta_{3}=\left(c_{0}-d_{0}\right)^{3}+\left(a_{2} c_{0}-a_{0} c_{2}\right)\left(c_{1}-d_{1}\right)^{3}+\cdots, \\
& \Delta_{3}^{\prime}=\left(c_{0}-d_{0}\right)^{3}+\left(a_{2} d_{0}-a_{0} d_{2}\right)\left(c_{1}-d_{1}\right)^{3}+\cdots .
\end{aligned}
$$

* Salmon, Higher Plane Curves, Dublin, 1897, pp. 191-192.

$\dagger$ Throughout this article the subscript on the symbol for an invariant indicates its weight. 
Each of these forms is transformed into a function of itself by the differential operators of $\$ 10$.

Evidently there are functional relations between the twelve relative invariants which these methods yield, since it has been shown that there can be but seven functionally independent invariants. This redundance makes unnecessary the use of the complicated $S$ and $T$ invariants of $\Delta$ and $\Delta^{\prime}$. The $S$ invariant of $\Delta-\Delta^{\prime}$ is quite simple however, and will be used.

13. A complete system of invariants. It will be shown that the following three covariants and seven invariants form a complete system:

$$
\begin{gathered}
\Lambda, \Lambda^{\prime}, \theta \equiv \Gamma-\Gamma^{\prime}, H_{2} \equiv \text { hessian of } \Gamma, H_{2}{ }^{\prime} \equiv \text { hessian of } \Gamma^{\prime}, \\
S_{4} \equiv S \text { of } \Delta-\Delta^{\prime}, \Lambda_{1}, \Gamma_{2}, \Gamma_{2}{ }^{\prime}, \Delta_{3} .
\end{gathered}
$$

Since we have only ten relations in fifteen variables, it is sufficient to show that they are independent when five of these variables are put equal to constants. It is found convenient to set $a_{0}=a_{1}=c_{2}=d_{2}=0$ and $a_{2}=1$. In fact this normalization can be made upon $\Gamma, \Gamma^{\prime}, \Delta, \Delta^{\prime}$ before $\mathrm{H}_{2}, \mathrm{H}_{2}{ }^{\prime}$, and $S_{4}$ are calculated. The ten invariants $(20)$ then reduce to the fairly simple forms:

$$
\begin{aligned}
\Lambda= & 3 x_{0}+\left(b_{2}+d_{1}\right) x_{2} \\
\Lambda^{\prime}= & 3 x_{0}+\left(b_{2}+c_{1}\right) x_{2} \\
\Theta= & -\left(c_{1}-d_{1}\right) x_{1}^{2}-b_{2}\left(c_{1}-d_{1}\right) x_{2}^{2}-2\left(c_{1}-d_{1}\right) x_{0} x_{2}, \\
H_{2}= & 4 c_{1}\left(d_{1}^{2}-b_{2} d_{1}+b_{2}^{2}\right)+12 b_{0} c_{1}-3 b_{1}^{2}-6 b_{1}\left(c_{0}+d_{0}\right) \\
& -3\left(c_{0}+d_{0}\right)^{2} \\
H_{2}^{\prime}= & 4 d_{1}\left(c_{1}^{2}-b_{2} c_{1}+b_{2}^{2}\right)+12 b_{0} d_{1}-3 b_{1}^{2}-6 b_{1}\left(c_{0}+d_{0}\right) \\
& -3\left(c_{0}+d_{0}\right)^{2}, \\
S_{4}= & \left(c_{1}-d_{1}\right)^{2} / 81\left[3 b_{2}\left(c_{0}-d_{0}\right)^{2}+6\left(c_{0}-d_{0}\right)\left(c_{1} d_{0}-c_{0} d_{1}\right)\right. \\
& \left.+3 b_{1}\left(c_{0}-d_{0}\right)\left(c_{1}-d_{1}\right)-\left(c_{1}-d_{1}\right)^{2}\left(b_{2}^{2}+3 b_{0}\right)\right] \\
\Lambda_{1}= & 3\left(c_{0}-d_{0}\right) \\
\Gamma_{2}= & 3\left(c_{0}-d_{0}\right)^{2}-c_{1}\left(c_{1}-d_{1}\right)^{2}, \\
\Gamma_{2}^{\prime}= & 3\left(c_{0}-d_{0}\right)^{2}-d_{1}\left(c_{1}-d_{1}\right)^{2}, \\
\Delta_{3}= & \left(c_{0}-d_{0}\right)^{3}+c_{0}\left(c_{1}-d_{1}\right)^{3}-c_{1}\left(c_{0}-d_{0}\right)\left(c_{1}-d_{1}\right)^{2} .
\end{aligned}
$$

14. Independence of the invariants. To prove the independence of these ten invariants it is sufficient to prove that the jacobian

$$
\frac{\partial\left(\Lambda, \Lambda^{\prime}, \theta, H_{2}, H_{2}^{\prime}, S_{4}, \Lambda_{1}, \Gamma_{2}, \Gamma_{2}^{\prime}, \Delta_{3}\right)}{\partial\left(x_{0}, x_{1}, x_{2}, b_{0}, b_{1}, b_{2}, c_{0}, c_{1}, d_{0}, d_{1}\right)}
$$

does not vanish identically. Now only the first three of these polynomials (21) involve $x_{0}, x_{1}, x_{2}$, and only the first six involve $b_{0}, b_{1}, b_{2}$. Hence it follows from considering the Laplace development that the jacobian (22) factors into three factors, viz., 


$$
\frac{\partial\left(\Lambda, \Lambda^{\prime}, \theta\right)}{\partial\left(x_{0}, x_{1}, x_{2}\right)} \times \frac{\partial\left(H_{2}, H_{2}^{\prime}, S_{4}\right)}{\partial\left(b_{0}, b_{1}, b_{2}\right)} \times \frac{\partial\left(\Lambda_{1}, \Gamma_{2}, \Gamma_{2}^{\prime}, \Delta_{3}\right)}{\partial\left(c_{0}, c_{1}, d_{0}, d_{1}\right)}
$$

It is sufficient to show that no one of these three jacobians vanishes identically in $c_{1}$, and hence it is sufficient to show that the coefficient of the highest power of $c_{1}$ in each jacobian is not identically zero. It is then permissible to drop all terms in each element except those involving $c_{1}$ to the highest power to which it occurs in that element; for the other terms evidently cannot enter into the term of highest degree in $c_{1}$ in the expansion of the determinant. It is important to be sure, however, that the coefficient of the highest power does not vanish, for this method does not give the coefficients of lower powers correctly. By this method it is easy to show that

$$
\begin{aligned}
& \frac{\partial\left(\Lambda, \Lambda^{\prime}, \theta\right)}{\partial\left(x_{0}, x_{1}, x_{2}\right)}=6 x_{1} c_{1}^{2}+\text { lower powers of } c_{1} \\
& \frac{\partial\left(H_{2}, H_{2}{ }^{\prime}, S_{4}\right)}{\partial\left(b_{0}, b_{1}, b_{2}\right)}=-\frac{16}{9} d_{1}\left(c_{0}-d_{0}\right) c_{1}{ }^{5}+\text { lower powers of } c_{1}, \\
& \frac{\partial\left(\Lambda_{1}, \Gamma_{2}, \Gamma_{2}{ }^{\prime}, \Delta_{3}\right)}{\partial\left(c_{0}, c_{1}, d_{0}, d_{1}\right)}=-9 c_{1}{ }^{7}+\text { lower powers of } c_{1} .
\end{aligned}
$$

Then the jacobian (22) becomes

$$
96 x_{1} d_{1}\left(c_{0}-d_{0}\right) c_{1}{ }^{14}+\text { lower powers of } c_{1},
$$

and the ten polynomials (20) are functionally independent and form a complete system of invariants and covariants of the linear algebra (14) from the standpoint of Lie.

\section{Characterization By invariants of a canonical Form}

15. The rank covariant. Let us consider the algebra in three units $1, e_{1}, e_{2}$ whose general number and multiplication table are given by (13) and (14) respectively. The rank* of every such algebra is three or two according as every number does not or does satisfy a quadratic equation. This rank is an arithmetic invariant under every linear transformation of units (15). It will be shown that for this example the arithmetic rank invariant can be replaced by a rational covariant.

* There is an equation $\rho(\omega)=0$ of lowest degree having $X$ as a right-hand (left-hand) root. The degree of this equation is called the right-hand (left-hand) rank of the algebra. (Cf. Dickson, Linear Algebras, p. 23.) It can be shown that there is at least one number satisfying no equation of lower degree. 
In every algebra of rank 3 with a principal unit, there is some number $x$ which does not satisfy a quadratic equation. Then the powers $1, x, x^{2}$ are linearly independent and may be taken as the units $1, e_{1}, e_{2}$. That is, the multiplication table of every rank 3 algebra in these units has the form (14) with $a_{0}=a_{1}=0, a_{2}=1$. Conversely, every algebra of this form is of rank 3 . Evidently, then, a necessary and sufficient condition that an algebra (14) be of rank 3 is that it be possible to make the above normalization.

From equations (16) it is seen that the conditions on a transformation (15) which shall make $a_{0}{ }^{\prime}=a_{1}{ }^{\prime}=0$ and $a_{2}{ }^{\prime}=1$ are

$$
\begin{aligned}
& A+D^{-1}\left(\alpha_{2} \beta_{0}-\alpha_{0} \beta_{2}\right) B+D^{-1}\left(\alpha_{0} \beta_{1}-\alpha_{1} \beta_{0}\right) C=0 \\
& D^{-1} \beta_{2} B-D^{-1} \beta_{1} C=0 \\
& -D^{-1} \alpha_{2} B+D^{-1} \alpha_{1} C=1
\end{aligned}
$$

These conditions are readily found to be equivalent to

$$
\beta_{0}=A, \quad \beta_{1}=B, \quad \beta_{2}=C,
$$

where $A, B, C$, given in (16), are polynomials in the $\alpha$ 's and the constants of multiplication. Thus the $\alpha$ 's can be chosen arbitrarily and the $\beta$ 's calculated from the above relations, provided only that the determinant of the transformation

$$
D=a_{2} \alpha_{1}^{3}+\left(-a_{1}+c_{2}+d_{2}\right) \alpha_{1}^{2} \alpha_{2}+\left(b_{2}-c_{1}-d_{1}\right) \alpha_{1} \alpha_{2}{ }^{2}-b_{1} \alpha_{2}{ }^{3}
$$

be different from zero. Now $\alpha_{1}$ and $\alpha_{2}$ can evidently be chosen so that $D$ is not zero unless every coefficient of $D$ vanishes. Then a necessary and sufficient condition that the algebra be of rank 3 is that not every coefficient $a_{2}$ etc. of (24) vanish.

It will now be shown that there exists a covariant $\Phi$ whose coefficients are the coefficients of (24). From (17) we form the absolute covariant

$$
\Gamma^{\prime}-\Gamma+\frac{1}{3}\left(\Lambda^{2}-\Lambda^{\prime 2}\right)
$$

every term of which involves $\left(c_{1}-d_{1}\right)$ or $\left(c_{2}-d_{2}\right)$. It was shown in (18) that $\left(c_{0}-d_{0}\right),\left(c_{1}-d_{1}\right),\left(c_{2}-d_{2}\right)$ are transformed cogrediently apart from the factor $D^{-1}$ with $x_{0}, x_{1}, x_{2}$, so we may substitute $x_{1}$ and $x_{2}$ respectively for these expressions and obtain a relative invariant of weight -1 ,

$$
\Phi \equiv a_{2} x_{1}^{3}+\left(-a_{1}+c_{2}+d_{2}\right) x_{1}{ }^{2} x_{2}+\left(b_{2}-c_{1}-d_{1}\right) x_{1} x_{2}{ }^{2}-b_{1} x_{2}{ }^{3},
$$

whose coefficients are the coefficients of (24). Hence a necessary and sufficient condition that an algebra (14) be of rank 3 is that $\Phi$ does not vanish identically. 
16. Determination of a canonical form. We shall designate as the generic case that case for which neither $\Phi, \Gamma_{2}-\Gamma_{2}{ }^{\prime}$, nor $\Lambda-\Lambda^{\prime}$ vanishes identically, and reduce this generic case by rational transformations in the general field $F$ to a canonical form-i. e., to a form in which each remaining coefficient is a relative invariant under the most general transformation which does not destroy the normalization. Since $\Phi$ is not identically zero, the multiplication table can at once be reduced as in $\S 15$. Then from (23) we see that in order to preserve this normalization the $\beta$ 's must obey the conditions:

$$
\begin{aligned}
& \beta_{0}=\alpha_{0}^{2}+\alpha_{2}^{2} b_{0}+\alpha_{1} \alpha_{2}\left(c_{0}+d_{0}\right) \\
& \beta_{1}=\alpha_{2}^{2} b_{1}+2 \alpha_{0} \alpha_{1}+\alpha_{1} \alpha_{2}\left(c_{1}+d_{1}\right) \\
& \beta_{2}=\alpha_{1}^{2}+\alpha_{2}^{2} b_{2}+2 \alpha_{0} \alpha_{2}+\alpha_{1} \alpha_{2}\left(c_{2}+d_{2}\right)
\end{aligned}
$$

If $c_{2}-d_{2}$ is not already zero, it is possible to effect a transformation making $c_{2}{ }^{\prime}-d_{2}{ }^{\prime}$ equal to zero. From (16) it is seen that under every transformation,

$$
c_{2}^{\prime}-d_{2}^{\prime}=-\alpha_{2}\left(c_{1}-d_{1}\right)+\alpha_{1}\left(c_{2}-d_{2}\right) .
$$

Therefore if we choose $\alpha_{1}$ and $\alpha_{2}$ satisfying the conditions

$$
\begin{gathered}
\alpha_{1}\left(c_{2}-d_{2}\right)=\alpha_{2}\left(c_{1}-d_{1}\right), \\
D=\alpha_{1}{ }^{3}+\left(c_{2}+d_{2}\right) \alpha_{1}{ }^{2} \alpha_{2}+\left(b_{2}-c_{1}-d_{1}\right) \alpha_{1} \alpha_{2}{ }^{2}-b_{1} \alpha_{2}{ }^{3} \neq 0,
\end{gathered}
$$

and the $\beta$ 's according to (25), we have the required transformation. Multiplying the second equation of $(27)$ by $\left(c_{2}-d_{2}\right)^{3}$ and eliminating $\alpha_{1}$ by means of the first equation, we have

$$
\begin{aligned}
D=\alpha_{1}{ }^{3}\left[\left(c_{1}-d_{1}\right)^{3}+\right. & \left(c_{2}+d_{2}\right)\left(c_{2}-d_{2}\right)\left(c_{1}-d_{1}\right)^{2} \\
& \left.+\left(b_{2}-b_{1}-c_{1}-d_{1}\right)\left(c_{2}-d_{2}\right)^{3}\right] .
\end{aligned}
$$

Hence this normalization is possible if and only if the expression in brackets is different from zero. But this expression is precisely the reduced form of the invariant $\Gamma_{2}^{\prime}-\Gamma_{2}$ which we have assumed not zero.

Since the covariant

$$
\Lambda-\Lambda^{\prime}=\left(c_{2}-d_{2}\right) x_{1}-\left(c_{1}-d_{1}\right) x_{2}
$$

does not vanish identically for this case, we know that $c_{1}-d_{1}$ is not zero. From (26) it is then seen that a necessary and sufficient condition on a transformation that shall leave this normalization undisturbed is that $\alpha_{2}=0$. Then conditions (25) require that $\alpha_{1} \neq 0, \alpha_{2}=0, \beta_{0}=\alpha_{0}{ }^{2}, \beta_{1}=2 \alpha_{0} \alpha_{1}, \beta_{2}=\alpha_{1}{ }^{2}$, and hence $D=\alpha_{1}{ }^{3}$. We see from (16) that by (15)

$$
c_{2}^{\prime}=3 \alpha_{0} \alpha_{1}+\alpha_{1}^{2} c_{2},
$$


so let us now apply the transformation

$$
\left(\begin{array}{ccc}
\alpha_{0} & \alpha_{1} & \alpha_{2} \\
\beta_{0} & \beta_{1} & \beta_{2}
\end{array}\right)=\left(\begin{array}{ccc}
-c_{2} / 3 & 1 & 0 \\
c_{2}^{2} / 9 & -2 c_{2} / 3 & 1
\end{array}\right)
$$

which reduces $c_{2}{ }^{\prime}$ to zero. Dropping accents, we obtain the canonical form of the generic case for rational transformations, viz.,

$$
\begin{aligned}
& e_{1}{ }^{2}=e_{2} \text {, } \\
& e_{2}^{2}=b_{0}+b_{1} e_{1}+b_{2} e_{2} \\
& e_{1} e_{2}=c_{0}+c_{1} e_{1} \text {, } \\
& e_{2} e_{1}=d_{0}+d_{1} e_{1}, \quad c_{1}-d_{1} \neq 0 \text {. }
\end{aligned}
$$

It is evident from (28) that for $c_{2}=0$ and $\alpha_{1} \neq 0$, a necessary and sufficient condition that $c_{2}{ }^{\prime}=0$ is that $\alpha_{0}=0$. Then the most general transformation which will leave (29) unaltered in form is of the type

$$
\left(\begin{array}{ccc}
0 & \alpha_{1} & 0 \\
0 & 0 & \alpha_{1}^{2}
\end{array}\right), D=\alpha_{1}^{3}
$$

The effect of this transformation on the coefficients of (29) is to make

$$
\begin{aligned}
b_{0}^{\prime} & =\alpha_{1}^{4} b_{0}, \quad b_{1}^{\prime}=\alpha_{1}^{3} b_{1}, \quad b_{2}^{\prime}=\alpha_{1}^{2} b_{2}, \quad c_{0}^{\prime}=\alpha_{1}^{3} c_{0}, \quad c_{1}^{\prime}=\alpha_{1}^{2} c_{1}, \\
d_{0}{ }^{\prime} & =\alpha_{1}{ }^{3} d_{0}, \quad d_{1}^{\prime}=\alpha_{1}{ }^{2} d_{1} .
\end{aligned}
$$

Hence no further reduction by rational transformations is possible, although by a transformation in general irrational any one of these coefficients which is not zero can be reduced to unity. Then the other six are parameters which cannot be altered except by a factor which is a root of unity.

17. Characterization of parameters. It is seen from (30) that the most that any transformation can do to (29), preserving its form, is to multiply each coefficient by a power of the determinant. It is natural to expect then that each coefficient in (29) is the reduced form of a relative invariant of the original form (14). We shall expect $c_{0}, d_{0}$ and $b_{1}$ to be relative invariants of weight $1 ; c_{1}, d_{1}$ and $b_{2}$ of weight $\frac{2}{3}$; and $b_{0}$ of weight $\frac{4}{3}$. We prove that this is so by actually finding these invariants in terms of known invariants. The invariants which characterize $c_{0}, c_{1}, d_{0}, d_{1}$ will be denoted by, respectively, $C^{0}, C^{\prime}, D^{0}, D^{\prime}$.

Making the normalization indicated in (29), we find that the invariants (19) reduce to the forms

$$
\begin{aligned}
& \Lambda_{1}=\Lambda_{1}{ }^{\prime}=3\left(c_{0}-d_{0}\right) \\
& \Gamma_{2}=3\left(c_{0}-d_{0}\right)^{2}-c_{1}\left(c_{1}-d_{1}\right)^{2} \\
& \Gamma_{2}{ }^{\prime}=3\left(c_{0}-d_{0}\right)^{2}-d_{1}\left(c_{1}-d_{1}\right)^{2} \\
& \Delta_{3}=\Delta_{3}{ }^{\prime}=\left(c_{0}-d_{0}\right)^{3}+c_{0}\left(c_{1}-d_{1}\right)^{3}-c_{1}\left(c_{0}-d_{0}\right)\left(c_{1}-d_{1}\right)^{2} .
\end{aligned}
$$


We have exactly four relations to determine four unknowns. We find $c_{0}$ in terms of the reduced invariants (31) and define $C^{0}$ to be the corresponding function of the complete invariants, so that $C^{0}$ is an invariant which reduces to $c_{0}$ under the normalization (29). Similarly we find invariants which reduce to the other parameters. Thus, since $\Gamma_{2}{ }^{\prime}-\Gamma_{2} \neq 0$,

$$
\begin{aligned}
& C^{0}=\frac{\Delta_{3}-\frac{1}{27} \Lambda_{1}{ }^{3}+\frac{1}{3} \Lambda_{1}\left(\frac{1}{3} \Lambda_{1}{ }^{2}-\Gamma_{2}\right)}{\Gamma_{2}{ }^{\prime}-\Gamma_{2}}, \\
& D^{0}=\frac{\Delta_{3}-\frac{1}{27} \Lambda_{1}{ }^{3}+\frac{1}{3} \Lambda_{1}\left(\frac{1}{3} \Lambda_{1}{ }^{2}-\Gamma_{2}{ }^{\prime}\right)}{\Gamma_{2}{ }^{\prime}-\Gamma_{2}}, \\
& C^{\prime}=\frac{\frac{1}{3} \Lambda_{1}{ }^{2}-\Gamma_{2}}{\left(\Gamma_{2}{ }^{\prime}-\Gamma_{2}\right)^{\frac{2}{3}}}, \quad D^{\prime}=\frac{\frac{1}{3} \Lambda_{1}{ }^{2}-\Gamma_{2}{ }^{\prime}}{\left(\Gamma_{2}{ }^{2}-\Gamma_{2}\right)^{\frac{3}{3}}}
\end{aligned}
$$

Evidently the weights are as predicted.

18. Equations defining the remaining parameters. Let us now consider the problem of finding invariants which characterize $b_{0}, b_{1}, b_{2}$. In addition to the invariants (31) we use the hessians $H_{2}$ and $H_{2}{ }^{\prime}$ of $\Gamma$ and $\Gamma^{\prime}$, and the invariants $S_{4}$ and $T_{6}$ of $\Delta-\Delta^{\prime}$. Under the normalization (29) we find
(a) $H_{2}, \quad$ (b) $H_{2}{ }^{\prime}, \quad$ (c) $S_{4}$ as given by (21),
(d) $T_{6}=4 l^{2} m p^{3}-3 l^{2} r^{2} p^{2}+6 p^{2} l q r s-12 m r p q^{3}+8 q^{3} r^{3}-27 p^{2} q^{2} s^{2}$,

where

$$
\begin{aligned}
& l=c_{0}-d_{0}, m=b_{0}\left(c_{1}-d_{1}\right)-b_{1}\left(c_{0}-d_{0}\right), p=\frac{-1}{3}\left(c_{1}-d_{1}\right), \\
& q=\frac{-1}{3}\left(c_{1}-d_{1}\right), s=\frac{1}{3}\left[b_{2}\left(c_{0}-d_{0}\right)+2 c_{1} d_{0}-2 c_{0} d_{1}\right], \\
& r=\frac{-1}{3} b_{2}\left(c_{1}-d_{1}\right) .
\end{aligned}
$$

Making use of the relation (33) in (32c), we have (34):

$$
\begin{aligned}
& H_{2}-H_{2}^{\prime}=4\left(c_{1}-d_{1}\right)\left(b_{2}^{2}+3 b_{0}-c_{1} d_{1}\right) \\
& b_{1}\left(c_{0}-d_{0}\right)\left(c_{1}-d_{1}\right)+b_{2}\left(c_{0}-d_{0}\right)^{2}=\frac{1}{3}\left[81 S_{4} /\left(c_{1}-d_{1}\right)\right. \\
& \left.-6\left(c_{0}-d_{0}\right)\left(c_{1} d_{0}-c_{0} d_{1}\right)+\frac{1}{4}\left(c_{1}-d_{1}\right)\left(H_{2}-H_{2}^{\prime}\right)+c_{1} d_{1}\left(c_{1}-d_{1}\right)^{2}\right]
\end{aligned}
$$

Multiplying (32b) by $c_{1}$ and subtracting this product from the product of $(32 b)$ by $d_{1}$, we obtain

$$
\begin{aligned}
3 b_{1}^{2} & +6 b_{1}\left(c_{0}+d_{0}\right)+4 b_{2} c_{1} d_{1}=\left(d_{1} H_{2}-c_{1} H_{2}{ }^{\prime}\right) /\left(c_{1}-d_{1}\right) \\
& +4 c_{1} d_{1}\left(c_{1}+d_{1}\right)-3\left(c_{0}+d_{0}\right)^{2} .
\end{aligned}
$$


Directly from (33) comes

$$
b_{2}^{2}+3 b_{0}=\frac{1}{\left(4 c_{1}-4 d_{1}\right)}\left[H_{2}-H_{2}^{\prime}+4 c_{1} d_{1}\left(c_{1}-d_{1}\right)\right]
$$

Now $c_{0}, d_{0}, c_{1}, d_{1}$ are the values which the known invariants $C^{0}, D^{0}, C^{\prime}, D^{\prime}$ take when the general algebra becomes (29). Consider functions $B^{0}, B^{\prime}, B^{\prime \prime}$ of the coefficients of the general algebra such that $B^{0}$ has the value $b_{0}, B^{\prime}$ the value $b_{1}$ and $B^{\prime \prime}$ the value $b_{2}$ when the general algebra becomes (29). Then corresponding to (34), (35), (36), and (32d), we have

$$
\begin{aligned}
& B^{\prime}\left(C^{0}-D^{0}\right)\left(C^{\prime}-D^{\prime}\right)+B^{\prime \prime}\left(C^{0}-D^{0}\right)=E, \\
& 3 B^{\prime 2}+6 B^{\prime}\left(C^{0}+D^{0}\right)+4 B^{\prime \prime} C^{\prime} D^{\prime}=F, \\
& B^{\prime \prime 2}+3 B^{0}=G, \\
& T_{6}=4 l^{2} m p^{3}-3 l^{2} r^{2} p^{2}+6 p^{2} l q r s-12 m r p q^{3}+8 q^{3} r^{3}-27 p^{2} q^{2} s^{2},
\end{aligned}
$$

where

$$
\begin{aligned}
& E= \frac{1}{3}\left[81 S_{4} /\left(C^{\prime}-D^{\prime}\right)^{2}-6\left(C^{0}-D^{0}\right)\left(C^{\prime} D^{0}-C^{0} D^{\prime}\right)\right. \\
&\left.+\frac{1}{4}\left(C^{\prime}-D^{\prime}\right)\left(H_{2}-H_{2}{ }^{\prime}\right)+C^{\prime} D^{\prime}\left(C^{\prime}-D^{\prime}\right)^{2}\right] \\
& F=\left(D^{\prime} H_{2}-C^{\prime} H_{2}\right) /\left(C^{\prime}-D^{\prime}\right)+4 C^{\prime} D^{\prime}\left(C^{\prime}+D^{\prime}\right)-3\left(C^{0}+D^{0}\right)^{2} \\
& G= \frac{1}{\left(4 C^{\prime}-4 D^{\prime}\right)}\left[H_{2}-H_{2}^{\prime}+4 C^{\prime} D^{\prime}\left(C^{\prime}-D^{\prime}\right)\right], \\
& l= C^{0}-D^{0}, \quad m=B^{0}\left(C^{\prime}-D^{\prime}\right)-B^{\prime}\left(C^{0}-D^{0}\right), \\
& p=-\frac{1}{3}\left(C^{\prime}-D^{\prime}\right), \quad q=-\frac{1}{3}\left(C^{\prime}-D^{\prime}\right), \\
& r=-\frac{1}{3} B^{\prime \prime}\left(C^{i}-D^{\prime}\right), \quad s=\frac{1}{3}\left[B^{\prime \prime}\left(C^{0}-D^{0}\right)+2 C^{\prime} D^{0}-2 C^{0} D^{\prime}\right] .
\end{aligned}
$$

Equations (37) determine the invariants $B^{0}, B^{\prime}, B^{\prime \prime}$ uniquely. A practical method for determining them is to calculate the two solutions of the first three equations of (37) and retain only the one which satisfies the fourth relation.

Thus the invariants of the linear algebra (14) which we have found are sufficient to isolate and characterize the generic case.

The University of Chicago,

Chicago, Ilt. 